\title{
Padrões temporais na assembléia de peixes na gamboa do Sucuriú, Baía de Paranaguá, Brasil
}

\author{
Henry Louis Spach ${ }^{1}$, Cesar Santos ${ }^{1,2} \&$ Rodrigo Santiago Godefroid ${ }^{1}$ \\ ${ }^{1}$ Centro de Estudos do Mar, Universidade Federal do Paraná. Caixa Postal 50002, 83255-000, Pontal do Paraná, Paraná, \\ Brasil. E-mail: hlspach@cem.ufpr.br \\ ${ }^{2}$ Curso de Pós-graduação em Zoologia, Departamento de Zoologia, Universidade Federal do Paraná. Caixa Postal 19020, \\ 81531-980 Curitiba, Paraná, Brasil.
}

\begin{abstract}
Temporal patterns of the fish assembly in the Sucuriú tidal creek, Paranaguá Bay, Brazil. The ichthyofauna of a tidal creek in Rasa da Cotinga Island, Paranaguá Bay, Paraná was studied through monthly collections in the quadrature high and low tides, with seine net of $30 \mathrm{~m} \mathrm{x} 1.5 \mathrm{~m}$ and $10 \mathrm{~mm}$ mesh and "fyke" net of $30 \mathrm{~m} \mathrm{x} 1.5 \mathrm{~m}$ with $10 \mathrm{~mm}$ mesh in the wings and $8 \mathrm{~mm}$ mesh in the bag. Together with the nekton sampling, temperature, salinity and water transparency values were also obtained as well as current speed. A total of 9068 fish belonging to 54 species and 23 families were collected. The ichthyofauna was dominated by Harengula clupeola (Cuvier, 1829), Atherinella brasiliensis (Quoy \& Gaimard, 1825), Sphoeroides greeley (Gilbert, 1900) and Sphoeroides testudineus (Linnaeus, 1758), that contributed to $66.1 \%$ of the capture in number and $79.7 \%$ in weight. Statistical differences between the collection months and months groups were observed for the number of species, number and weight of fish, species richness and species diversity (weight). The observed temporal biological structure seems to be better explained by the rainfall, rainfall and salinity and combined action of the rainfall, salinity and water transparency.
\end{abstract}

KEY WORDS. Fish fauna, seasonal variation, mangrove.

As associações de peixes estuarinos são usualmente caracterizadas por constituintes transientes de espécies marinhas, de água doce ou anádromas, que usam os estuários como áreas de alimentação, de criação de larvas e juvenis, ou em parte de suas migrações para a reprodução. Residentes permanentes, que completam todo o seu ciclo de vida dentro dos estuários, estão representados por poucas espécies, vivendo principalmente em águas rasas (DAY et al. 1989, KNEIB 1997).

A grande e variada oferta de recursos alimentares, principalmente devido à alta produção primária e a conseqüente produção secundária adequada como alimento para juvenis de peixes (Robertson \& Blaber 1992), o aumento de refúgios contra predação resultante da complexidade estrutural, pouca profundidade, turbidez e o reduzido número de peixes carnívoros de grande porte (RoberTson \& Blaber 1992, Mullin 1995), são os principais fatores que explicam a alta abundância de peixes em áreas de manguezais.

Cortando os manguezais existe uma complexa rede de canais de maré (gamboas), bastante heterogêneas quanto à profundidade e o regime de maré, que na baixa-mar concentram os juvenis de peixe e na preamar possibilitam o movimento dos peixes para dentro da floresta do mangue (VANCE $e t$ al. 1996, Ronnback et al. 1999). A função ecológica e a compo- sição das assembléias de peixes em canais de maré em marisma são bem conhecidas (Weinstein \& BRooks 1983, RountreE \& Able 1992, Allen et al. 1994), por outro lado, em manguezais, principalmente da costa brasileira, o conhecimento sobre a utilização dos canais de maré por peixes é reduzido (CoRrêA et al. 1988, Barletta 1999, Vendel et al. 2002).

Neste estudo, foram feitas observações sobre a variação temporal na abundância e estrutura da assembléia de peixes em um canal de maré da Ilha Rasa da Cotinga, situada em uma zona da Baía de Paranaguá sob influência direta da massa de água costeira presente na plataforma continental adjacente. Um biótopo intimamente associado a manguezal, com condições ambientais diferentes das áreas estuarinas abertas, e que pode apresentar diferenças no padrão de ocupação por peixes.

\section{MATERIAL E MÉTODOS}

\section{Área de estudo}

O presente estudo foi desenvolvido na gamboa do Sucuriú, localizada na Ilha Rasa da Cotinga $\left(25^{\circ} 32^{\prime} 24.385^{\prime \prime}\right.$ S e $48^{\circ} 24^{\prime} 53.33^{\prime \prime} \mathrm{W}$ ) situada no setor euhalino da Baía de Paranaguá, Paraná. Esta região apresenta clima do tipo pluvial temperado 
(Cfa), com chuvas durante todo o ano e maiores pluviosidades e temperaturas ocorrendo no verão (MAACK 1981). A pluviosidade e a ingressão da cunha salina afetam a salinidade, que possui sazonalidade nítida. $\mathrm{O}$ oxigênio dissolvido apresenta concentração média estável e próxima a $6 \mathrm{ml} / \mathrm{l}$ (KNOPPERs et al. 1987). Os regimes de vento na direção leste e sudeste são predominantes, chegando a atingir $6 \mathrm{~m} / \mathrm{s}$. A maré apresenta desigualdades diurnas e segue um padrão semidiurno (KNopPers et al. 1987). A gamboa do Sucuriú é um canal meândrico com aproximadamente $2 \mathrm{Km}$ de extensão, profundidade variando entre 0,5 e $8 \mathrm{~m}$. A região de entrada possui alto hidrodinamismo, que decresce nas partes mais internas. Além disso, apresenta sedimento variando entre silte grosso e areia muito fina pobremente selecionada e vegetação composta por marismas e manguezais.

\section{Desenho amostral}

A coletas de peixes foram realizadas no período diurno mensalmente entre agosto de 1998 e julho de 1999, usando redes tipo picaré e Fyke. Esta última trata-se de um amostrador passivo, desenhado para utilizar a dinâmica da maré para coletar o nécton existente em gamboas. A rede tipo picaré $(30$ x 1,5 $\mathrm{m}$, malha de $10 \mathrm{~mm}$ ), foi utilizada nos arrastos feitos na margem da gamboa no estofo da primeira baixa-mar (Fig. 1, Pontos P1 a P4) e durante o estofo da segunda baixa-mar (Fig. 1, Pontos P5 a P8). A rede tipo Fyke é composta por duas asas (comprimento: $30 \mathrm{~m}$; altura: 1,5 m, malha: $10 \mathrm{~mm}$ ) e um saco (malha: $8 \mathrm{~mm}$ ). Em cada momento amostral, duas redes "Fyke" foram instaladas nos pontos " $\mathrm{A}$ " em um canal lateral e " $\mathrm{B}$ " na margem do canal principal, com a boca da rede voltada para a entrada do canal, com despesca no estofo da preamar, sendo transferidas para os pontos $\mathrm{C}$ e $\mathrm{D}$ na margem do canal principal, onde permaneceram pescando com a boca da rede posicionada na direção interna do canal de maré, até o final da baixa-mar (Fig. 1). Em conjunto com a amostragem do nécton, de hora em hora, durante a preamar (na entrada do Sucuriú) e baixa-mar (na porção mediana do Sucuriú) foram obtidos dados de temperatura e salinidade com STD, da velocidade das correntes com um correntômetro e de transparência da água com um disco de Secchi. Os dados de pluviosidade foram obtidos junto à estação meteorológica do Centro de Estudos do Mar da Universidade Federal do Paraná, localizada a cerca de 6 $\mathrm{Km}$ da área de estudo. Após a identificação dos peixes, foram obtidos dados de peso (g), comprimento padrão e total (mm) e, quando possível, foi determinado o sexo.

\section{Análise de Dados}

As amostras de todas as redes foram agrupadas por mês de coleta. As possíveis diferenças temporais nos parâmetros ambientais e no número de espécies, número de peixes, biomassa, riqueza de espécies estimada pelo índice de Margalef, da diversidade de espécies (número e peso) estimada pelo índice de Shannon - Wiener e de eqüitabilidade estimada pelo índice de eqüitabilidade de Pielou (Ludwig \& Reynolds 1988), foram examinadas através de um modelo de ANOVA fixo (SOKAL $\&$ Rohlf 1995), aplicado aos dados transformados pela raiz quarta e testados quanto à homogeneidade da variância (teste de Bartelett) e de normalidade da distribuição (prova de KolmorovSmirnov). Se o resultado da ANOVA indicava a existência de diferenças estatísticas ( $\mathrm{p}<0,01 ; \mathrm{p}<0,05)$ aplicou-se, o teste da Mínima Diferença Significativa (LSD) para determinar quais médias foram significativamente diferentes. Quando qualquer

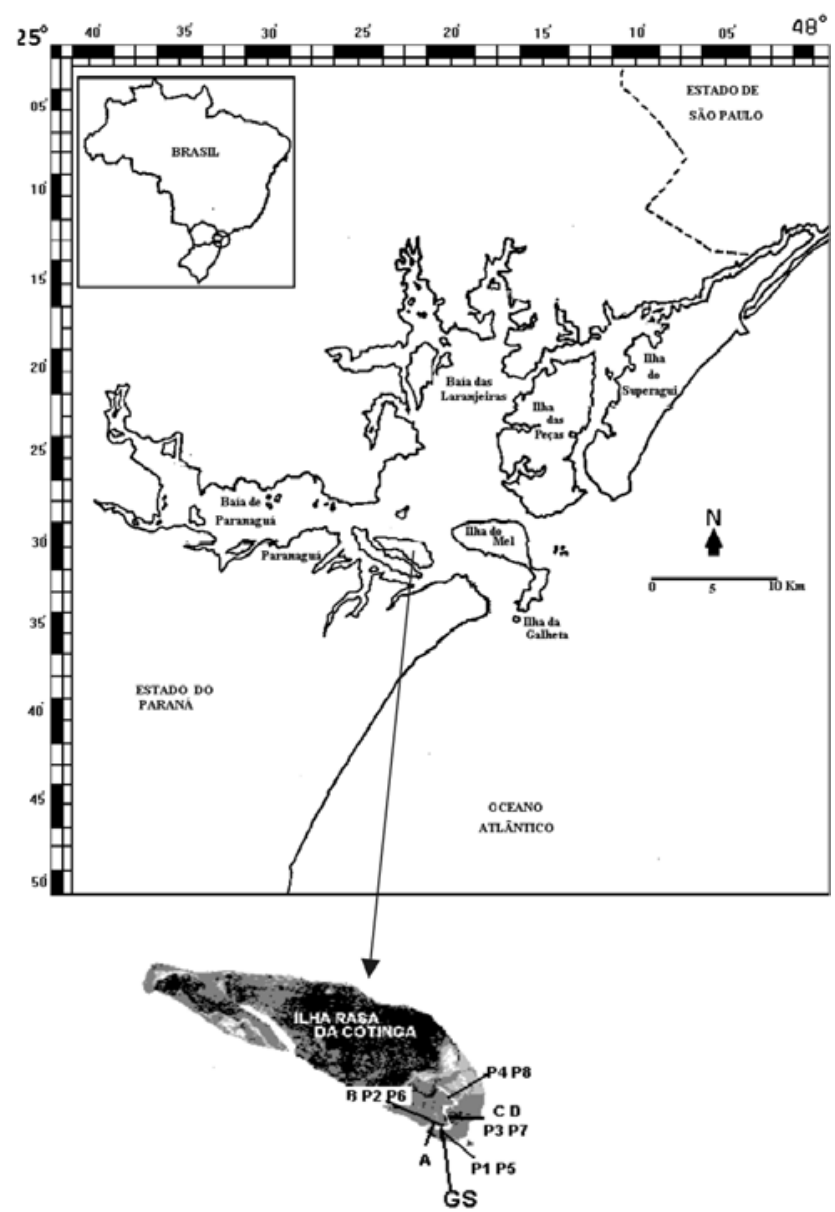

Figura 1. Mapa do litoral paranaense, com a localização do ponto de amostragem na Gamboa do Sucuriú (GS) na llha Rasa da Cotinga com a rede tipo picaré (P1 a P8) e com a rede tipo "fyke" ("A" a "D").

pressuposto da ANOVA não era atendido, as comparações foram feitas através do teste não paramétrico de Kruskal - Wallis (Conover 1990, Sokal \& Rohlf 1995).

Os índices de Riqueza de espécies de Margalef, Diversidade de Shannon - Wiener (número e peso) e de Eqüitabilidade de Pielou foram calculados através do pacote estatístico PRIMER versão 4.0 (Plymounth Marine Laboratory, Inglaterra). As análises multivariadas, Cluster (método normal) e MDS não métrico, deste pacote estatístico foram utilizadas para avaliar a variação temporal na composição e abundância das espécies capturadas. Antes das análises, foram selecionadas as espécies mais freqüentes, com contribuição nunca inferior a $1 \%$ da captura total e as mais constantes, ou seja, que foram encontradas em no mínimo seis meses do ano. Além disso, os valores de ocorrência numérica dessas espécies foram transformados pela raiz quarta e o coeficiente de similaridade de Bray-Curtis foi usado para a produção da matriz de similaridade, com os grupos sendo fusionados pela média simples dos seus valores de similaridade (UPGMA). 

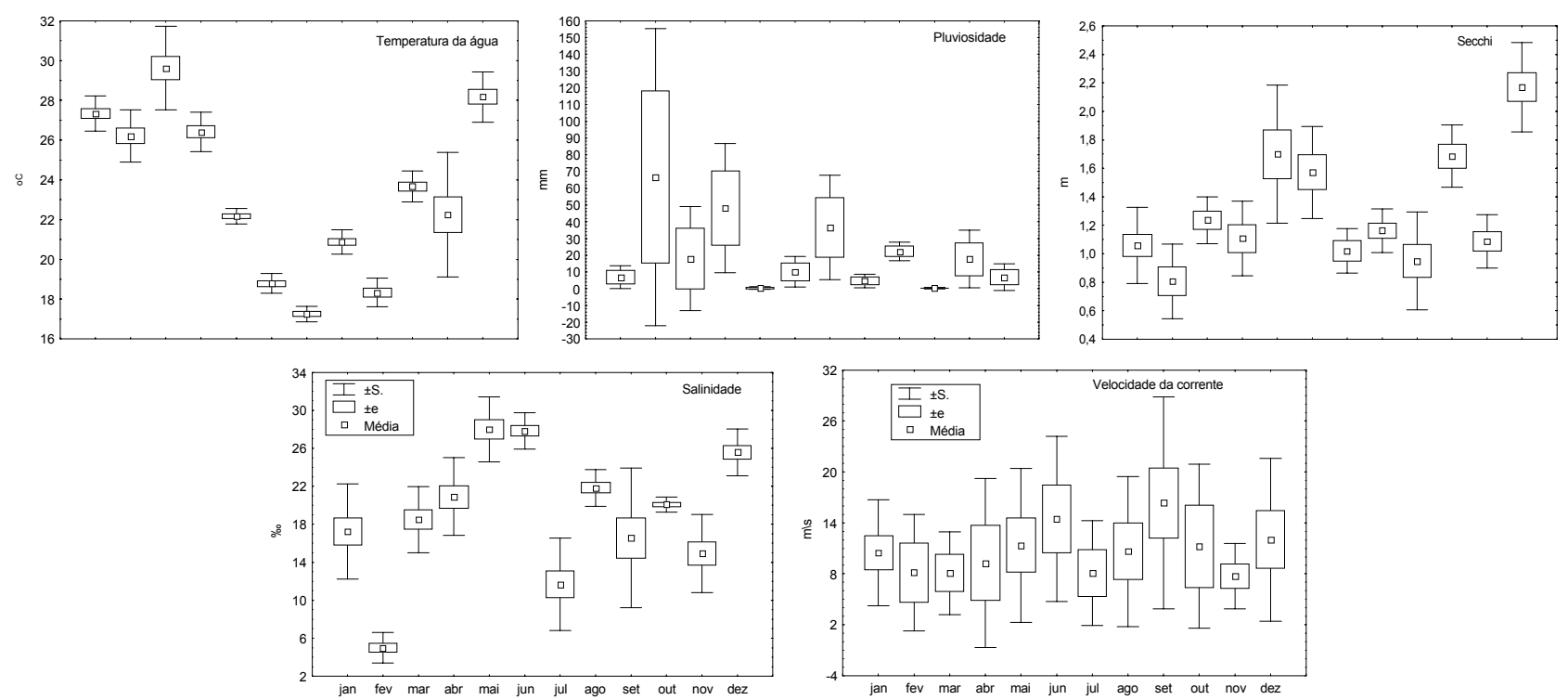

Figura 2. Variação mensal na média da temperatura da água, pluviosidade, salinidade, transparência da água (Secchi) e velocidade da corrente, durante o período de amostragem na Gamboa do Sucuriú.

A análise BIOENV permitiu relacionar os dados bióticos com os dados abióticos, revelando quais os parâmetros mais importantes para a estruturação do padrão de distribuição temporal observado na composição e abundância de espécies. Isto é feito através da simples comparação multivariada da concordância entre as duas matrizes de similaridade de dados bióticos e abióticos. Para os dados biológicos, a similaridade utilizada foi a de Bray-Curtis, e para os abióticos a distância Euclidiana normalizada. A similaridade das percentagens (SIMPER) permitiu indicar quais espécies foram as principais responsáveis pelas similaridades dentro de cada grupo, definido no Cluster (método normal) (espécies mais comuns) e para a dissimilaridade entre esses grupos (espécies mais discriminantes). Esta análise de similaridade nada mais faz que decompor as similaridades entre grupos e calcular a contribuição de cada espécie através de sua ocorrência relativa em cada grupo (Clarke \& WARWICK 1994). As estações do ano foram definidas da seguinte forma: setembro a novembro = primavera; dezembro a fevereiro $=$ verão; março a maio = outono; junho a agosto $=$ inverno .

\section{RESULTADOS}

\section{Parâmetros ambientais}

A temperatura da água apresentou médias significativamente menores em junho, julho e setembro, com valores médios intermediários em maio, agosto, outubro e novembro e maiores de dezembro até abril (Fig. 2; $\mathrm{H}=100,06 \mathrm{p}=0,00$ ). Apesar das diferenças significativas, nenhuma tendência sazonal foi observada para a pluviosidade, com picos de precipitação média em fevereiro e abril, e menores em maio, agosto e outubro (Fig. 2; F=2,46 p=0,03). A água apresentou as menores salinidades médias em fevereiro e julho e maiores em abril, maio e dezembro e valores intermediários e pouco diferentes entre si nos demais meses do ano (Fig. 2; $\mathrm{H}=50,63 \mathrm{p}=0,00$ ). A menor e a maior transparência média ocorreram na gamboa em fevereiro e dezembro, respectivamente. Nos meses de maio, junho e outubro a transparência foi significativamente maior do que nos demais meses, exceção a dezembro. As transparências médias de janeiro, março, abril, julho, agosto, setembro e novembro não diferiram estatisticamente (Fig. 2; $F=13,02 \mathrm{p}=$ $0,00)$. Não foram significativas as diferenças entre as velocidades médias de corrente (Fig. 2; $F=0,46 \mathrm{p}=0,92$ ).

\section{Ictiofauna}

Um total de 9068 peixes, na sua maioria formas imaturas, pertencentes a 54 espécies, foi registrado nas capturas mensais realizadas durante um ano (Tab. I). Das 23 famílias encontradas na área, Carangidae (sete espécies) e Gerreidae (seis espécies) foram as mais diversas. O nécton amostrado foi dominado por Harengula clupeola, Atherinella brasiliensis, Sphoeroides greeley e Sphoeroides testudineus, com cada uma dessas espécies contribuindo com mais de $7,5 \%$ da captura total de peixes. As espécies $S$. testudineus $(32,9 \%)$ e $S$. greeley $(17,0 \%)$ foram dominantes e assim como A. brasiliensis, foram pescadas em todos os meses de coleta.

\section{Variação temporal}

O número médio de espécies foi maior em fevereiro, março e abril, com valores intermediários em maio e setembro, e menores e não diferentes estatisticamente entre si nos demais meses (Fig. 3, Tab. II). Observa-se um aumento da captura média em número de peixes entre a metade da primavera e o verão, com acentuada diminuição até a metade do inverno, seguindo-se um período com poucas oscilações. Excetuando-se os meses de janeiro, maio e junho, a captura média foi significativamente menor em julho, já as capturas de março e abril só foram significativamente maiores do que as de novem- 
Tabela I. Composição específica e abundância de peixes nas capturas mensais na Gamboa do Sucuriú (\% da captura total).

\begin{tabular}{|c|c|c|c|c|c|c|c|c|c|c|c|c|c|}
\hline Espécies & Jan & Fev & Mar & Abr & Mai & Jun & Jul & Ago & Set & Out & Nov & Dez & $\%$ \\
\hline Anchoa lyolepis (Evermann \& Marsh, 1902) & 0 & 29 & 0 & 0 & 0 & 0 & 0 & 0 & 0 & 0 & 0 & 0 & 0,3 \\
\hline Anchoa parva (Meek \& Hildebrand, 1923) & 0 & 0 & 0 & 0 & 27 & 0 & 2 & 0 & 0 & 0 & 0 & 0 & 0,3 \\
\hline Anchoa tricolor (Steindachner, 1880) & 0 & 4 & 40 & 8 & 19 & 2 & 2 & 0 & 0 & 0 & 0 & 4 & 0,9 \\
\hline Cetengraulis edentulus (Cuvier, 1829) & 0 & 108 & 0 & 0 & 0 & 0 & 0 & 0 & 0 & 0 & 0 & 0 & 1,2 \\
\hline Lycengraulis grossidens (Agassiz, 1828) & 0 & 1 & 0 & 2 & 0 & 0 & 0 & 0 & 1 & 0 & 0 & 9 & 0,1 \\
\hline Harengula clupeola (Cuvier, 1829) & 0 & 35 & 76 & 491 & 12 & 0 & 3 & 0 & 11 & 0 & 0 & 134 & 8,4 \\
\hline Opisthonema oglinum (Lesueur, 1818) & 0 & 295 & 5 & 0 & 0 & 0 & 0 & 0 & 0 & 0 & 0 & 0 & 3,3 \\
\hline Sardinella brasiliensis (Steindachner, 1879) & 0 & 13 & 74 & 8 & 0 & 0 & 0 & 0 & 0 & 0 & 0 & 4 & 1,1 \\
\hline Genidens genidens (Valenciennes, 1840) & 0 & 0 & 0 & 0 & 65 & 0 & 0 & 0 & 3 & 0 & 1 & 0 & 0,8 \\
\hline Synodus foetens (Linnaeus, 1766) & 0 & 1 & 0 & 2 & 0 & 0 & 0 & 0 & 0 & 0 & 0 & 0 & 0,0 \\
\hline Mugil curema (Valenciennes, 1836) & 2 & 8 & 2 & 6 & 25 & 0 & 2 & 0 & 50 & 1 & 1 & 0 & 1,1 \\
\hline Mugil gaimardianus (Desmarest, 1831) & 0 & 0 & 2 & 0 & 0 & 0 & 0 & 50 & 1 & 0 & 0 & 1 & 0,6 \\
\hline Mugil liza (Valenciennes, 1836) & 0 & 0 & 0 & 0 & 0 & 0 & 0 & 0 & 0 & 0 & 1 & 0 & 0,0 \\
\hline Mugil platanus (Günther, 1880) & 0 & 0 & 1 & 0 & 0 & 0 & 0 & 0 & 0 & 0 & 0 & 0 & 0,0 \\
\hline Mugil sp. & 9 & 3 & 19 & 1 & 2 & 0 & 0 & 0 & 1 & 1 & 3 & 1 & 0,4 \\
\hline Atherinella brasiliensis (Quoy \& Gaimard, 1825) & 87 & 77 & 115 & 44 & 57 & 9 & 11 & 46 & 99 & 79 & 26 & 55 & 7,8 \\
\hline Strongylura marina (Walbaum, 1792) & 0 & 0 & 0 & 0 & 0 & 0 & 0 & 0 & 0 & 4 & 1 & 1 & 0,1 \\
\hline Strongylura sp. & 0 & 1 & 0 & 0 & 0 & 0 & 0 & 0 & 0 & 0 & 0 & 0 & 0,0 \\
\hline Hyporhamphus unifasciatus (Ranzani, 1842) & 0 & 0 & 2 & 0 & 1 & 0 & 0 & 0 & 0 & 0 & 0 & 0 & 0,0 \\
\hline Poecilia vivipara (Bloch \& Schneider, 1801) & 33 & 11 & 13 & 304 & 0 & 12 & 4 & 32 & 22 & 14 & 47 & 8 & 5,5 \\
\hline Centropomus parallelus (Poey, 1860) & 0 & 1 & 0 & 12 & 2 & 21 & 0 & 18 & 3 & 0 & 6 & 5 & 0,7 \\
\hline Centropomus undecimalis (Bloch, 1792) & 0 & 0 & 4 & 2 & 0 & 0 & 0 & 2 & 2 & 2 & 6 & 1 & 0,2 \\
\hline Centropomus sp. & 0 & 0 & 0 & 0 & 0 & 4 & 0 & 0 & 0 & 0 & 0 & 0 & 0,0 \\
\hline Diplectrum radiale (Quoy \& Gaimard, 1824) & 0 & 0 & 0 & 2 & 0 & 0 & 1 & 0 & 0 & 0 & 0 & 0 & 0,0 \\
\hline Caranx hippos (Linnaeus, 1766) & 1 & 0 & 0 & 0 & 0 & 0 & 0 & 0 & 0 & 0 & 0 & 0 & 0,0 \\
\hline Caranx latus (Agassiz, 1831) & 35 & 0 & 2 & 0 & 0 & 0 & 0 & 0 & 1 & 0 & 0 & 0 & 0,4 \\
\hline Oligoplites saurus (Bloch \& Schneider, 1801) & 2 & 3 & 13 & 16 & 1 & 0 & 0 & 0 & 0 & 0 & 0 & 0 & 0,4 \\
\hline Selene vomer (Linnaeus, 1758) & 0 & 1 & 0 & 0 & 0 & 0 & 0 & 0 & 1 & 0 & 0 & 0 & 0,0 \\
\hline Trachinotus falcatus (Linnaeus, 1758) & 0 & 0 & 7 & 1 & 0 & 0 & 0 & 0 & 0 & 0 & 0 & 0 & 0,1 \\
\hline Trachinotus sp. & 0 & 1 & 0 & 0 & 5 & 0 & 0 & 0 & 0 & 0 & 0 & 0 & 0,1 \\
\hline Uraspis secunda (Poey, 1860) & 0 & 2 & 0 & 1 & 0 & 0 & 0 & 0 & 0 & 0 & 0 & 0 & 0,0 \\
\hline Lutjanus griseus (Linnaeus, 1758) & 0 & 0 & 1 & 0 & 0 & 0 & 0 & 0 & 0 & 0 & 0 & 0 & 0,0 \\
\hline Diapterus rhombeus (Cuvier, 1830) & 0 & 27 & 107 & 44 & 62 & 11 & 3 & 32 & 50 & 0 & 8 & 4 & 3,8 \\
\hline Eucinostomus argenteus (Baird \& Girard, 1855) & 0 & 24 & 120 & 24 & 16 & 6 & 19 & 41 & 30 & 3 & 7 & 3 & 3,2 \\
\hline Eucinostomus gula (Cuvier, 1830) & 4 & 4 & 27 & 5 & 12 & 0 & 4 & 10 & 11 & 10 & 9 & 24 & 1,3 \\
\hline Eucinostomus melanopterus (Bleeker, 1863) & 3 & 0 & 2 & 7 & 5 & 9 & 14 & 6 & 13 & 39 & 22 & 2 & 1,3 \\
\hline Eucinostomus sp. & 73 & 164 & 2 & 4 & 4 & 64 & 0 & 0 & 0 & 0 & 0 & 0 & 3,4 \\
\hline Eugerres brasilianus (Cuvier, 1830) & 0 & 0 & 0 & 0 & 0 & 0 & 0 & 1 & 0 & 0 & 0 & 0 & 0,0 \\
\hline Ulaema lefroyi (Goode, 1874) & 0 & 0 & 0 & 0 & 0 & 0 & 0 & 1 & 0 & 0 & 0 & 0 & 0,0 \\
\hline Bairdiella ronchus (Valenciennes, 1830) & 1 & 0 & 0 & 0 & 0 & 0 & 0 & 0 & 0 & 1 & 0 & 5 & 0,1 \\
\hline
\end{tabular}


Tabela I. Continuação.

\begin{tabular}{|c|c|c|c|c|c|c|c|c|c|c|c|c|c|}
\hline Espécies & Jan & Fev & Mar & Abr & Mai & Jun & Jul & Ago & Set & Out & Nov & Dez & $\%$ \\
\hline Guavina guavina (Valenciennes, 1837) & 0 & 0 & 0 & 0 & 0 & 0 & 0 & 0 & 1 & 1 & 0 & 0 & 0,0 \\
\hline Bathygobius soporator (Valenciennes, 1837) & 4 & 3 & 2 & 5 & 5 & 0 & 1 & 1 & 4 & 0 & 4 & 2 & 0,3 \\
\hline Chaetodipterus faber (Broussonet, 1782) & 0 & 2 & 1 & 3 & 2 & 1 & 0 & 0 & 0 & 0 & 0 & 0 & 0,1 \\
\hline Scomberomorus cavalla (Cuvier, 1829) & 0 & 11 & 0 & 0 & 0 & 0 & 0 & 0 & 0 & 0 & 0 & 0 & 0,1 \\
\hline Citharichthys arenaceus (Evermann\&Marsh,1900) & 6 & 3 & 4 & 14 & 0 & 4 & 3 & 1 & 3 & 3 & 7 & 2 & 0,6 \\
\hline Citharichthys spilopterus (Günther, 1862) & 10 & 8 & 5 & 27 & 8 & 8 & 11 & 3 & 5 & 2 & 0 & 0 & 1,0 \\
\hline Citharichthys sp. & 0 & 6 & 2 & 4 & 0 & 3 & 0 & 0 & 0 & 0 & 0 & 0 & 0,2 \\
\hline Etropus crossotus (Jordan \& Gilbert, 1882) & 0 & 1 & 2 & 1 & 0 & 0 & 0 & 0 & 0 & 0 & 1 & 0 & 0,1 \\
\hline Achirus lineatus (Linnaeus, 1758) & 1 & 0 & 2 & 0 & 0 & 0 & 0 & 0 & 0 & 0 & 0 & 0 & 0,0 \\
\hline Lagocephalus laevigatus (Linnaeus, 1766) & 0 & 1 & 0 & 0 & 1 & 0 & 0 & 0 & 0 & 0 & 0 & 0 & 0,0 \\
\hline Sphoeroides greeley (Gilbert, 1900) & 86 & 82 & 95 & 123 & 67 & 83 & 53 & 241 & 114 & 242 & 212 & 145 & 17,0 \\
\hline Sphoeroides testudineus (Linnaeus, 1758) & 201 & 179 & 428 & 424 & 119 & 170 & 68 & 332 & 201 & 343 & 202 & 314 & 32,9 \\
\hline Sphoeroides tyleri (Shipp, 1972) & 0 & 0 & 0 & 0 & 0 & 0 & 0 & 8 & 0 & 0 & 0 & 0 & 0,1 \\
\hline Cyclichthys spinosus (Linnaeus, 1758) & 2 & 0 & 2 & 5 & 5 & 7 & 2 & 5 & 0 & 2 & 1 & 0 & 0,3 \\
\hline
\end{tabular}
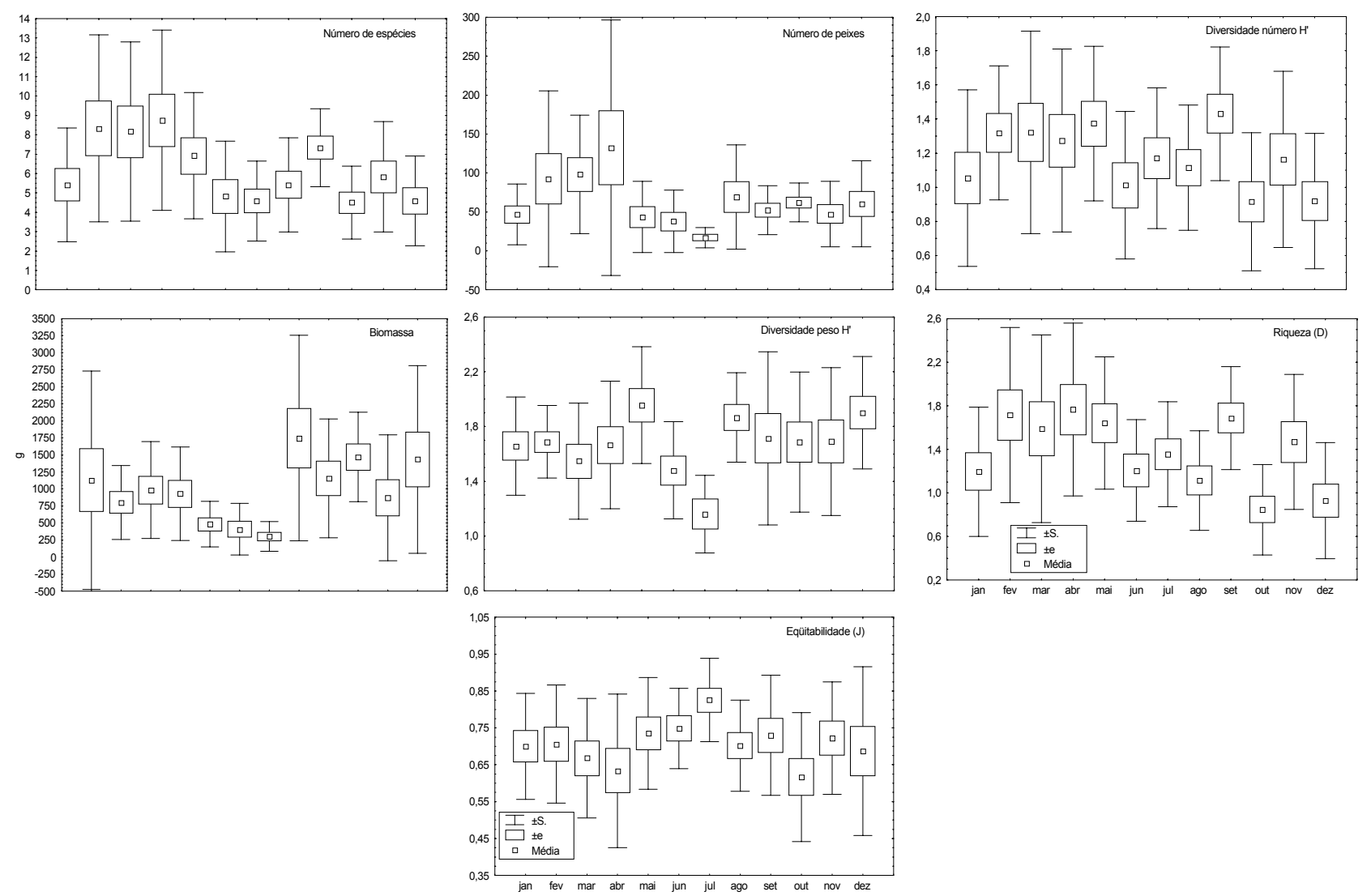

Figura 3. Variação mensal na média do número de espécies, número de peixes, biomassa e dos índices de riqueza de Margalef, diversidade de Shannon - Wiener e eqüitabilidade de Pielou na Gamboa do Sucuriú. 
Tabela II. Resultado da Análise de Variância (F) e do teste de Kruskal-Wallis (H), avaliando o efeito do mês de coleta e dos grupos de meses definidos no Cluster (modo Q), sobre a captura média em número de espécies, número de peixes, biomassa, riqueza de espécies de Margalef, diversidade de Shannon - Wiener (número e peso) e eqüitabilidade de Pielou, na gamboa do Sucuriú. Antes das análises, os dados foram transformados pela raiz quarta. O teste da Mínima Diferença Significativa (LSD) foi aplicado para determinar quais médias eram significativamente diferentes ( ${ }^{*}$ diferença significativa ao nível de $p<0,01$, ** diferença significativa ao nível de $p<0,05$, NS diferença não significativa).

\begin{tabular}{|c|c|c|c|c|c|c|c|c|c|}
\hline \multirow{2}{*}{ Parâmetros } & \multicolumn{4}{|c|}{ Mês } & \multicolumn{4}{|c|}{ Grupo } & \multirow{2}{*}{ LSD } \\
\hline & $\mathrm{F}$ & $\mathrm{p}$ & $\mathrm{H}$ & $\mathrm{p}$ & $\mathrm{F}$ & $\mathrm{p}$ & $\mathrm{H}$ & $p$ & \\
\hline Número de espécies & 2,55 & $0,00^{*}$ & & & 6,47 & $0,00^{*}$ & & & $\mathrm{~B}>\mathrm{AC} \mathrm{Ja}$ \\
\hline Número de peixes & & & 8,00 & $0,53 \mathrm{NS}$ & & & 12,53 & $0,00^{*}$ & A B Ja Ja C \\
\hline Biomassa & & & 12,66 & $0,18 \mathrm{NS}$ & & & 13,04 & $0,00^{*}$ & $A>B>C A B C=$ Janeiro \\
\hline Riqueza & & & 26,36 & $0,00^{*}$ & & & 8,89 & $0,03^{* *}$ & $\mathrm{~B}>\mathrm{AC} \mathrm{Ja}$ \\
\hline Diversidade (número) & 1,74 & $0,07 \mathrm{NS}$ & & & 2,54 & $0,06 \mathrm{NS}$ & & & \\
\hline Diversidade (peso) & 2,14 & $0,02^{\star *}$ & & & 4,23 & $0,00^{*}$ & & & \\
\hline Eqüitabilidade & 1,35 & $0,20 \mathrm{NS}$ & & & & & 5,55 & $0,13 \mathrm{NS}$ & $A B>C A B C=J a$ \\
\hline
\end{tabular}
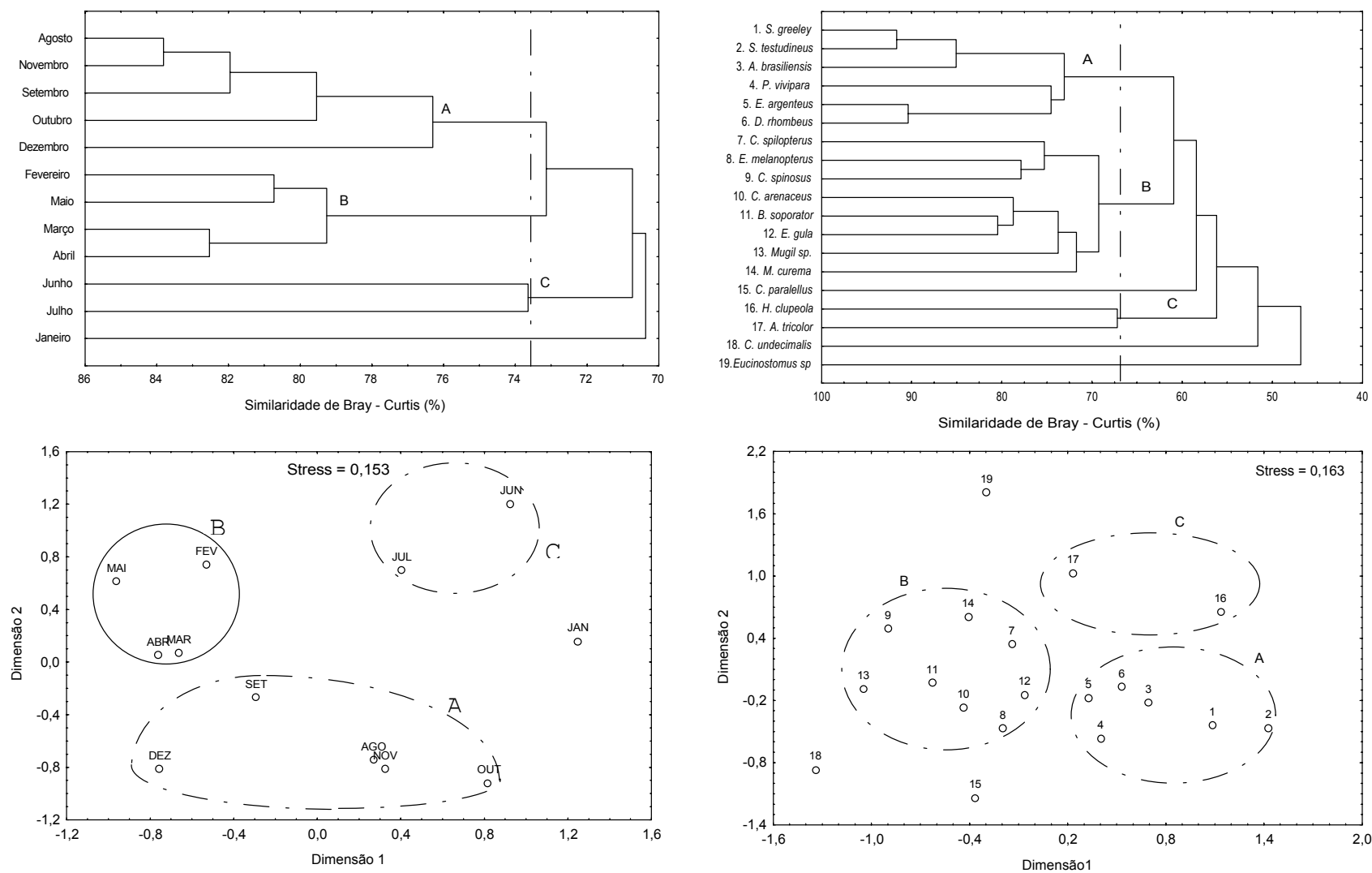

Figura 4. Dendrograma e ordenação pelo método MDS baseados nos dados de densidade dos vinte taxa principais, amostrados mensalmente na Gamboa do Sucuriú. Grupos de espécies delineados no nível de $73,5 \%$ de similaridade no dendograma, estão circundados no gráfico de ordenação. O stress da ordenação MDS $=0,153$.

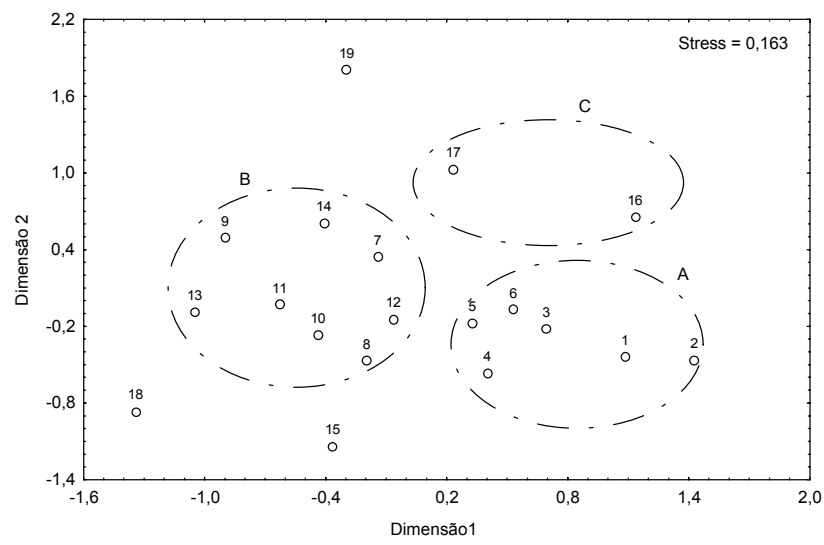

Figura 5. Dendograma e ordenação pelo método MDS mostrando as similaridades entre os taxa mais abundantes baseadas em sua ocorrência ao longo dos doze meses de amostragem na Gamboa do rio Sucuriú. Grupos de espécies delineados no nível de $66,5 \%$ de similaridade. No gráfico de ordenação as espécies são representadas pelos seus respectivos números fornecidos no dendograma.

Revista Brasileira de Zoologia 20 (4): 591-600, dezembro 2003 
Tabela III. Contribuição percentual (\%) das espécies mais abundantes e constantes, na Gamboa do Sucuriú, para a similaridade dentro dos grupo A (agosto, setembro, outubro, novembro e dezembro), do grupo $B$ (fevereiro, março, abril e maio), do grupo $C$ (junho e julho) e para a dissimilaridade entre esses grupos ("A" x "B", "A" x "C", "B" $\mathrm{x}$ "C").

\begin{tabular}{|c|c|c|c|}
\hline & A & B & C \\
\hline Similaridade média dentro dos grupos (\%) & 79,16 & 80,05 & 73,64 \\
\hline Anchoa tricolor & & 5,50 & \\
\hline Harengula clupeola & & 7,53 & \\
\hline Atherinella brasiliensis & 10,92 & 9,21 & \\
\hline Poecilia vivipara & 8,30 & & \\
\hline \multicolumn{4}{|l|}{ Centropomus parallelus } \\
\hline Diapterus rhombeus & & 8,39 & \\
\hline Eucinostomus argenteus & 6,36 & 7,16 & \\
\hline Eucinostomus gula & 7,62 & & \\
\hline Eucinostomus melanopterus & 6,67 & & \\
\hline \multicolumn{4}{|l|}{ Eucinostomus spp. } \\
\hline \multicolumn{4}{|l|}{ Citharichthys spilopterus } \\
\hline Sphoeroides greeley & 15,19 & 10,05 & 43,63 \\
\hline \multirow[t]{2}{*}{ Sphoeroides testudineus } & 16,91 & 12,27 & 18,08 \\
\hline & $A \times B$ & $A \times C$ & $\mathrm{~B} \times \mathrm{C}$ \\
\hline Dissimilaridade média entre os grupos (\%) & 26,86 & 29,67 & 28,76 \\
\hline Anchoa tricolor & 9,26 & 6,15 & 4,10 \\
\hline Harengula clupeola & 12,49 & & 12,63 \\
\hline Mugil curema & 6,46 & & \\
\hline Mugil spp. & & 5,34 & 7,83 \\
\hline Atherinella brasiliensis & & 5,96 & 6,26 \\
\hline Centropomus undecimalis & & 7,57 & \\
\hline Diapterus rhombeus & & & 6,44 \\
\hline Eucinostomus argenteus & & 3,09 & \\
\hline \multicolumn{4}{|l|}{ Eucinostomus gula } \\
\hline \multicolumn{4}{|l|}{ Eucinostomus melanopterus } \\
\hline Eucinostomus spp. & 10,94 & & 9,08 \\
\hline Sphoeroides greeley & 3,57 & 5,19 & \\
\hline Sphoeroides testudineus & 3,04 & 5,16 & \\
\hline
\end{tabular}

bro, janeiro, maio, junho e julho (Fig. 3, Tab. II). A captura média em peso diminuiu entre o verão e o inverno, com valores significativamente menores em junho e julho, voltando a subir já em agosto e oscilando durante a primavera. De um modo geral as diferenças não foram significativas do final do inverno até a metade do outono (Fig. 3, Tab. II).

Apesar das diferenças significativas, nenhuma tendência sazonal foi observada na riqueza de espécies, com as meno- res riquezas de agosto, outubro e dezembro significativamente menores somente em comparação a setembro e ao período entre fevereiro e maio, mas não diferindo significativamente em relação aos demais meses (Fig. 3, Tab. II). A diversidade em peso foi em média significativamente menor em julho em comparação ao resto do ano, exceção a março e junho, este último com uma diversidade média menor em relação às capturas de maio, agosto e dezembro. A diversidade (peso) não diferiu estatisticamente entre o verão, outono e primavera (Fig. 3, Tab. II). As capturas mensais não diferiram estatisticamente no que se refere a eqüitabilidade e diversidade em número (Tab. II).

Considerando-se a abundância mensal dos 19 taxa selecionados, a análise de Cluster (método normal), separou no nível de similaridade de 73,5 \% os meses de coleta em três grupos, deixando o mês de janeiro isolado (Fig. 4). O primeiro grupo "A", conectado no nível de similaridade de 76,3\%, contendo os meses de agosto, setembro, outubro, novembro e dezembro, período com a maior captura média em peso em relação ao resto do ano (Tab. II). Um segundo grupo "B", com similaridade de 79,3\%, composto por dois subgrupos, um no nível de $81,0 \%$ do dendograma, com os meses de fevereiro e maio, e o outro, com uma similaridade de $82,5 \%$, formado pelos meses de março e abril, meses com a maior captura média em número de espécies e riqueza (Tab. II). Por último, o grupo "C" com os meses de junho e julho ligados no nível de similaridade de 73,6 \%, quando ocorre as menores capturas médias em número de peixes, em peso e as menores diversidades (peso) médias (Tab. II). Os grupos definidos no Cluster (meses) também podem ser observados na plotagem bidimensional do MDS, com o grupo "A" ocupando a parte inferior, o grupo "B" acima à esquerda e o grupo "C" na parte superior direita. O stress de 0,153 indica que as distâncias gráficas nas duas dimensões não estariam representando adequadamente as similaridades originais (Fig. 4).

Através da análise de similaridade de percentagens (SIMPER) verificou-se uma similaridade média de 79,16 \% no grupo "A" definido pelo Cluster (método normal) sendo $A$. brasiliensis, Poecilia vivípara (Bloch \& Schneider, 1801), Eucinostomus argenteus (Baird \& Girard, 1855), Eucinostomus gula (Cuvier, 1830), Eucinostomus melanopterus (Bleeker, 1863), S. greeley e $S$. testudineus as espécies que mais contribuíram para esta similaridade. No grupo "B" a similaridade média foi maior (80,05 \%) devido principalmente as espécies Anchoa tricolor, $H$. clupeola, A. brasiliensis, Diapterus rhombeus (Cuvier, 1830), E. argenteus, $S$. greeley e $S$. testudineus. Uma menor similaridade média ocorre entre os meses do grupo "C" (73,64\%) com as espécies $S$. greeley e $S$. testudineus contribuindo com $61,71 \%$ desta similaridade (Tab. III). A dissimilaridade média foi maior entre os grupos " $\mathrm{A}$ " $\mathrm{x}$ " $\mathrm{C}$ " $(29,67 \%)$ contribuindo principalmente A. brasiliensis, Centropomus undecimalis (Bloch, 1792) e E. gula. Entre os grupos " $\mathrm{B}$ " $\mathrm{x}$ " $\mathrm{C}$ " observou-se a segunda maior dissimilaridade média $(28,76 \%)$ sendo mais responsáveis por esta diferença os taxa $H$. clupeola, A. brasiliensis, Mugil spp., D. rhombeus e Eucinostomus sp. A menor dissimilaridade média foi entre os grupos "A" x "B" (26,86\%) sendo A. tricolor, Mugil curema (Valenciennes, 1836) e $S$. greeley as espécies que mais contribuíram para esta dissimilaridade.

A análise de Cluster (método inverso) aplicada aos dezenove taxa principais mostrou três grupos unidos nível de similaridade de $66,5 \%$ (Fig. 5). O primeiro grupo "A", com similaridade de 73,0 \%, formado pelo subgrupo das espécies $S$. greeley, $S$. testudineus e A. brasiliensis, presentes o ano todo com 
as maiores abundâncias, e pelo subgrupo das espécies $P$. vivipara, $E$. argenteus e D. rhombeus, presentes praticamente todo o período amostral, porém com abundâncias menores que as espécies do outro subgrupo e ausentes em algumas coletas (Tab. I). No grupo "B" apresentando similaridade de 69,0 \% dois subgrupos de taxa, um conectado no nível de similaridade de 75,0 \%, composto pelas espécies Citharichthys spilopterus (Günther, 1862), E. melanopterus e Cyclichthys spinosus (Linnaeus, 1758) e o outro unido a $72,0 \%$ de similaridade, com os taxa Citharichthys arenaceus (Evermann \& Marsh, 1900), Bathygobius soporator (Valenciennes, 1837), E. gula, Mugil sp. e M. curema, presentes na gamboa em todas as estações do ano porem em quantidades menores que as das espécies do grupo "A" (Tab. I). Por último o grupo "C", conectado ao nível de similaridade de $67,5 \%$, com as espécies $H$. clupeola e $A$. tricolor presentes na área em maior quantidade do início do verão até o final do outono e esporádicas no resto do ano. Não agruparam, Centropomus parallelus (Poey, 1860), presente em todas as estações na área, mas mais abundante no outono e inverno, $C$. undecimalis, sempre em pouca quantidade na área porem mais freqüente do final do inverno ao início do verão e ausente das amostras entre janeiro e julho, exceção aos meses de março e abril e Eucinostomus $s p$. ausente das amostras entre a metade do inverno e início do verão (Tab. I). Os grupos presentes no nível de similaridade de $66,5 \%$ no dendograma podem ser vistos nas primeiras duas dimensões do MDS, com o grupo " $\mathrm{A}$ " posicionado na parte inferior direita, o grupo " $\mathrm{B}$ " mais à esquerda e o grupo " $\mathrm{C}$ " acima do grupo " $\mathrm{A}$ ". O stress de 0,163 reflete uma certa dificuldade para representar graficamente as similaridades entre as espécies (Fig. 5).

Ao analisar a influência individual ou conjunta dos parâmetros ambientais sobre a estruturação do padrão biológico, observou-se baixos valores de correlação. Parecem explicar melhor o padrão de ocorrência da ictiofauna a pluviosidade, a ação conjunta da pluviosidade e salinidade e da pluviosidade, salinidade e transparência da água (Tab. IV).

Tabela IV. Resultados mais significativos do BIOENV, indicando a influência da temperatura da água $(T)$, pluviosidade $(P)$, salinidade $(\mathrm{S})$, transparência da água (Tr) e velocidade da corrente $(\mathrm{V})$. Os valores são os coeficientes de correlação harmônico ponderado 'Spearm rank', para as variáveis isoladas e cada combinação. Os resultados somente indicam a natureza e a magnitude da correlação.

\begin{tabular}{cccc}
\hline$P$ & $S$ & $P S$ & $S T r$ \\
\hline$-0,315$ & $-0,273$ & $-0,315$ & $-0,236$ \\
$P \operatorname{Tr}$ & $P S T r$ & $P S T r V$ & TPSTRV \\
$-0,222$ & $-0,302$ & $-0,176$ & $-0,110$ \\
\hline
\end{tabular}

\section{DISCUSSÃO}

Apesar da grande diversidade de espécies, na gamboa do Sucuriú o domínio numérico foi exercido por um grupo pequeno de espécies, o que já foi observado em outras áreas da Baía de Paranaguá (Godefroid et al. 1997, Vendel et al. 2002). Três das espécies dominantes (H. clupeola, S. testudineus e $S$. greeleyi) nesta gamboa, também estiveram entre as mais abun- dantes na gamboa do Baguaçu, ambas situadas no setor eualino desta baía (Vendel et al. 2002). Por outro lado, na zona de arrebentação de uma praia situada na entrada da Baía de Paranaguá, a cerca de $5 \mathrm{Km}$ dos dois rios de maré, com exceção da $H$. clupeola, a dominância foi exercida por outras espécies (GODEFROID et al.1997).

Vendel et al. (2002) encontraram uma maior abundância de peixes na gamboa do Baguaçu no outono e parte do inverno, enquanto que na praia de Pontal do Sul os peixes foram mais numerosos no verão e parte do outono (GODEFroID et al.1997). Um maior quantidade de peixes estiveram presentes no Sucuriú do final do verão até a metade do outono, porém, a abundância de peixes foi baixa no inverno. Um maior número de indivíduos e espécies entre o final do verão e no outono estaria refletindo a utilização mais intensa neste período dos ambientes rasos do setor eualino da Baía de Paranaguá pelos peixes (Godefroid et al.1997, Vendel et al. 2002, este trabalho).

As medidas de diversidade são usualmente utilizadas para descrever as tendências temporais na estrutura das comunidades de peixes (Pielou 1969). No presente estudo, a riqueza específica (D) foi em média maior de fevereiro ao final do outono, e menor na primavera, um padrão temporal diferente do observado na gamboa do Baguaçu (Vendel et al. 2002). No Sucuriú a riqueza de espécies variou de 0,8 a 1,80, sendo estes valores menores do que os observados no Baguaçu $(1,69-3,75$, Vendel et al. 2002) e na praia de Pontal do Sul $(2,00-5,05$, GoDEFroId et al.1997).

A diversidade em número de exemplares $\left(\mathrm{H}^{\prime}\right)$ não variou significativamente no Sucuriú. A flutuação no número de peixes foi maior do que na biomassa dos peixes, mas ambos os parâmetros não apresentaram uma dinâmica sazonal definida. Entre agosto e dezembro observa-se uma menor diversidade em número em comparação a diversidade em peso, um reflexo da entrada na gamboa de um número maior de reprodutores adultos de poucas espécies. As diferenças entre as diversidades em número e peso são menores no outono, período em que os exemplares maiores migram para fora da área de amostragem e ocorre uma forte dominância de juvenis de poucas espécies. No Sucuriú o padrão de variação temporal e a amplitude do índice de diversidade em número $(1,1-1,95)$ foram diferentes dos observados no rio de maré do Baguaçu (0,06-1,76, VENDEL et al. 2002) e na zona de arrebentação da praia de Pontal do Sul (1,13-2,32, GodefroId et al.1997), o que indica a diferente utilização temporal desses ambientes pelos peixes.

Na gamboa estudada as associações de espécies definidas pelo padrão de ocorrência numérica, estão formadas por taxa residentes e periódicos (visitantes ocasionais e marinhas dependentes do estuário), na sua maioria, consumidores primários. Um grupo é constituído por quatro espécies estuarinas ( $S$. greeleyi, $S$. testudineus, A. brasiliensis e D. rhombeus), uma marinha dependente do estuário (E. argenteus) e outra de águas continentais (P. vivípara). Apesar de todas se alimentarem de invertebrados marinhos, parecem existir algumas condições que possibilitam a minimização da competição por recursos alimentares (Figueiredo \& Menezes 1978, 1980, Chaves \& Serenato 1998, Chaves \& Otto 1999, Figueiredo \& Menezes 2000) Considerando-se as diferenças anatômicas, provavelmente os baiacus $S$. greeleyi e $S$. testudineus se alimentam de presas maiores do que $E$. argenteus e D. rhombeus, seus competidores diretos pelos invertebrados bentônicos, fato importante principalmente para os juvenis destas espécies, uma vez que todas utilizam a gamboa

Revista Brasileira de Zoologia 20 (4): 591-600, dezembro 2003 
como área de criação na mesma época. Alem das influências da morfologia sobre a ecologia trófica dos peixes, enquanto $P$. vivipara se alimenta em águas com baixa salinidade, em especial na interface marginal onde ocorre percolação de água doce do lençol freático (KNeIB 1997), as espécies A. brasiliensis e S.greeleyi exerceriam uma maior pressão de predação na gamboa durante a preamar, quando as condições de salinidade são mais adequadas para estas espécies (Figueiredo \& Mentzes 1978, Prosdocimo \& Freire 2001).Entre as quatro espécies do grupo, provavelmente $S$. testudineus é a única espécie que não tem a sua área de ação limitada pela a salinidade, o que possibilitaria uma melhor exploração dos recursos alimentares disponíveis na gamboa (Prosdocimo \& Freire 2001).

O segundo grupo, que é constituído por cinco espécies marinhas dependentes de estuário (E. melanopterus, C. spinosus, B. soporator, E.gula e M. curema) e apenas duas estuarinas $(C$. spilopterus e C. arenaceus) (Figueiredo \& Menezes 1980, Menezes \& Figueiredo 1985, Figueiredo \& Menezes 2000), foi dominado por formas jovens, presentes em todas as estações do ano, sempre em pequenas quantidades. Mesmo existindo sobreposição no uso do ambiente, as pequenas densidades podem estar contribuindo para diminuir a competição por alimento. Alem disto, enquanto $C$. spilopterus, C. arenaceus e B. soporator comem crustáceos e peixes (Menezes \& Figueiredo 1985, Chaves \& Serenato 1998), entre as demais espécies marinhas dependentes de estuário, M. curema é pastador (Menezes \& Figueiredo 1985) e $E$. melanopterus, C. spinosus e E. gula se alimentam principalmente de pequenos invertebrados (Chaves \& Serenato 1998, Chaves $\&$ Отто 1999). Também se agruparam $H$. clupeola e $A$. tricolor, duas espécies planctófogas (Figueiredo \& MENEzes 1978), representadas na área apenas por indivíduos juvenis, presentes na preamar em alguns poucos agregados no outono.

Como a variação temporal na estrutura da comunidade de peixes foi definida principalmente pelo padrão de ocorrência de espécies preferencialmente associadas a águas mais salinas, presentes no ambiente estudado principalmente com a entrada da maré, observou-se a relação direta entre a variabilidade amostral com o aumento na transparência e salinidade da água e inversa com o incremento da pluviosidade. A estratégia de uso da gamboa, condicionada pela tolerância das espécies a determinadas condições ambientais limitantes, segue, principalmente processos de curta duração (marés), e suas condicionantes meteorológicas.

\section{REFERÊNCIAS BIBLIOGRÁFICAS}

Allen, E.A.; P.E. Fell; M.A. Peck; J. A. Gieg, C.R. GuthKe \& M.D. NewKIRK. 1994. Gut contents of common mummichogs, Fundulus heteroclitus L., in a restored impounded marsh and in natural reference marshes. Estuaries. 17 (2): 462-471.

BARLETTA, M. 1999. Seasonal changes of density, biomass and species composition of fishes in different habitats of the Caeté estuary (Northern Brazilian coast - east Amazon). Zentrum für Marine Tropenökologie - ZMT - Contribuiton 7, 115p

Chaves, P.T.C. \& G. Отто. 1999. The mangrove as a temporary habitat for fish: the Eucinostomus species at Guaratuba Bay,

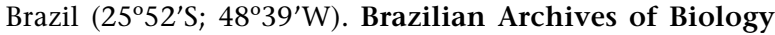
and Technology, Curitiba, 42 (1): 61-68.

Chaves, P.T.C. \& A. Serenato 1998. Diversidade de dietas na assembléia de linguados (Teleostei, Pleuronectiformes) do manguezal da Baía de Guaratuba, Paraná, Brasil. Revista Brasileira de Oceanografia, São Paulo, 46 (1): 61-68.

CLARKE, K.R. \& R.W. WARWICK. 1994. Change in marine communities: an aproach to statistical analysis and interpretation. Plymouth Marine Laboratory, 859p.

Conover, W.J. 1990. Pratical nonparametric statistics. New Jersey, Jhon Willey \& Sons, 584p.

Corrêa, M.F.M.; P.C.R. Cerdeiras \& J.C. Pieckzarka. 1988. Levantamento ictiológico do Rio Guanandi $\left(25^{\circ} 30^{\prime} 25^{\prime \prime} \mathrm{S}\right.$ e $45^{\circ} 45^{\prime}$ $50^{\prime \prime} \mathrm{W}$ ), sub-bacia do Rio Nhundiaquara (Morretes, PR, Brasil). Nerítica, Curitiba, 3 (1): 37-60.

Day Jr., J.W.; C.A.S. Hall; W.M. Kemp \& A. Yáñez-Arancibia. 1989. Estuarine Ecology. Nova York, John Wiley \& Sons, 558p.

Figueiredo, J.L. \& N. Menezes 1978. Manual de peixes marinhos do sudeste do Brasil. II. Teleostei (1). São Paulo, Museu de Zoologia da USP, 110p.

. 1980. Manual de peixes marinhos do sudeste do Brasil. III. Teleostei (2). São Paulo, Museu de Zoologia da USP, 90p.

2000. Manual de peixes marinhos do sudeste do Brasil. VI. Teleostei (5). São Paulo, Museu de Zoologia da USP, 116p.

Godefroid, R.S.; M. Hofstaetter, \& H.L. Spach. 1997. Structure of the fish assemblage in the surf zone beach at Pontal do Sul, Paraná. Nerítica, Curitiba, 11: 77-93.

KNeIB, R.T. 1997. The role of tidal marshes in the ecology of etuarine nekton. Oceanography and Marine Biology: an Annual Review, London, 34: 163-220.

Knoppers, B.A.; F.P. Brandini \& C.A. Thamm. 1987. Ecological studies in the Bay of Paranaguá. II Some physical and chemical characteristics. Nerítica, Curitiba, 2 (1): 1-36.

LudWIG, J.A. \& J.F. ReYNoLDs. 1988. Statistical ecology. New York, John Wiley \& Sons, 337p.

MAAK, R. 1981. Geografia física do Estado do Paraná. Rio de Janeiro, J. Olimpio Ed. Secr. Cultura e do Esporte do estado do Paraná, 450p.

Menezes, N. \& J.L. Figueiredo. 1985. Manual de peixes marinhos do sudeste do Brasil. V. Teleostei (4). São Paulo, Museu de Zoologia da USP, 90p.

Muluin, S.J. 1995. Estuarine fish populations among red mangrove prop roots of small overwash island. Wetlands, Lawrence, 15 (4): 324-329.

Pielou, E.C. 1969.The measurement of diversity in different types of biological colections. Journal of Theoretical Biology, New York, 13: 131-144.

Prosdocimo V. \& C.A. Freire. 2001. Ionic regulation in aglomerular tropical estuarine pufferfishes submitted to sea water dilution. Journal of Experimental Marine Biology and Ecology, Amsterdam, 262: 243-253.

RoberTSON, A.I. \& S.J.M. Blaber. 1992. Plankton, epibenthos and fish communities, p. 63-100. In: A.I. RoBERTSON \& D.M AlONGI (Eds). Tropical Mangrove Ecosystems (Coastal and Estuarine Studies 41). Washinhton, DC, American Geophysical Union, 236p.

Rönnbäck, P.; M. Troell; N. Kautsky \& J.H. Primavera. 1999. Distribution pattern of shrimps and fish among Avicennia and Rhizophora microhabitats in the Pagbilao mangroves, Philippines. Estuarine and Coastal Marine Science, London, 48: 223-234. 
RountreE, R.A. \& K.W. AbLe. 1992. Foraging habits, growth, and temporal patterns os salt marsh creek habit use by young of year summer flouder in New Jersey. Transactions of the American Fisheries Society, Bethesda, 121: 765-776.

Sokal, R.R. \& F.J. Rohlf. 1995. Biometry. New York, W.H. Freeman and Company, 859p.

Vance, D.J.; M.D.E. Haywood; D.S. Heales; R.A. Kenyon; N.R. Loneragan \& R.C. Pendrey. 1996. How far do prawns and fish move into mangroves? Distribution of juvenile banana prawns Penaeus merguiensis and fish in a tropical man- grove forest in northern Australia. Marine Ecology Progress Series, Oldendorf, 131: 115-124.

Vendel, A.L.; H.L. Spach; S.G. Lopes \& C. Santos. 2002. Structure and dynamics of fish assemblages in a tidal creek environment. Brazilian Archives of Biology and Technology, Curitiba, 45 (3): 365-373.

Weinstein, M.P. \& H.A. BRooKs. 1983. Comparative ecology of nekton residing in a tidal creek and adjacent seagrass meadow: community composition and structure. Marine Ecology Progress Series, Oldendorf, 12: 15-27.

Recebido em 09.01.2003; aceito em 14.X.2003.

Revista Brasileira de Zoologia 20 (4): 591-600, dezembro 2003 